$\begin{array}{llll}\text { Revista Latinoamericana de Medicina Conductual } & \text { Vol } 1 & \text { Num } 1 & 8-2010\end{array}$ Latin American Journal of Behavioral Medicine

César Garduño, Angélica Riveros y JuAn José SÁnchez-Sosa

Facultad de Psicología Universidad Nacional Autónoma de México

Recibido: Mayo 24, 2010

Revisado: Junio 16, 2010

Aceptado: Junio 28, 2010

\section{Calidad de Vida y Cáncer de Mama: Efectos de una Intervención Cognitivo-Conductual}

\author{
QUALITY OF LIFE AND BREAST CANCER:" EFFECTS OF A \\ COGNITIVE-BEHAVIORAL INTERVENTION
}

\begin{abstract}
La investigación objeto del presente artículo se financió con el proyecto IN-303908 del PAPIIT de la UNAM. César Garduño: recolección, captura y sistematización de datos, terapeuta, tesista, redacción de versión preliminar. A. Riveros: coordinación metodológica, análisis de datos, supervisión y corrección de informe. J. J. Sánchez Sosa, dirección de proyecto, planeación metodológica y logística, supervisión clínica, supervisión de informe final. Se agradece a: A. Martínez-Basurto, H. Cruz, G. Escorza, I. Moreno y C. Sierra su destacada labor como terapeutas, y a S. Alvarado A. por su apoyo institucional en el INCAN.

Dirigir correspondencia a: Correo: johannes@unam.mx
\end{abstract}

Resumen

El presente estudio examinó los efectos de una intervención Cognitivo-Conductual en la Calidad de Vida de pacientes con cáncer de mama. Estas pacientes experimentan sufrimiento por su condición médica y por los efectos colaterales de algunos procedimientos médicos y agudiza lo estresante de una condición económica. Participaron sesenta pacientes con diagnóstico confirmado de cáncer de mama no terminal. La principal medición incluyó el Inventario de Calidad de Vida y Salud (InCaViSa) con escalas sobre: preocupaciones, desempeño físico, aislamiento, percepción corporal, funciones cognitivas, actitud ante el tratamiento, tiempo libre, vida cotidiana, familia, redes sociales, dependencia y relación con el médico. Se usó un diseño pre-test, post-test y seguimientos a 1 y 3 meses. Después de cumplir con los requisitos éticos, se expuso individualmente a sesenta pacientes a un procedimiento de psico-educación. Posteriormente se las expuso a procedimientos para: establecer habilidades de adhesión terapéutica, identificar y modificar cogniciones disfuncionales y mejorar su autorregulación emocional. Los resultados revelaron mejorías estadística y clínicamente significativas en prácticamente todas las áreas para la gran mayoría de las participantes.

Palabras Clave: Cáncer de mama, calidad de vida, cognitivo-conductual.

\begin{abstract}
The present study examined the effects of a cognitive-behavioral intervention on the Quality of Life of breast cancer patients. These patients suffer from both their medical condition and the side-effects of its treatment, often complicated by dire socioeconomic conditions. A total of sixty non-terminal patients with confirmed diagnostic of breast cancer participated. Measurement included the Quality of Life and Health Inventory (InCaViSa) with scales on: worrying, physical performance, personal isolation, body perception, cognitive functioning, attitude toward medical treatment, recreation, family interaction, social networks/support, medical dependence and relationship with the health team. Measurement involved pretest, posttest and one and three months follow up. Once ethical requirements were met, sixty patients were individually exposed to a psycho-education module and then to procedures aimed at: establishing therapeutic adherence behaviors, changing distorted or dysfunctional cognitions, and improving emotional self regulation. Results revealed statistically and clinically relevant improvement in all Quality of Life Areas and self care for the great majority of participants.
\end{abstract}

Key words: Breast cancer, quality of life, cognitivebehavioral 
El cáncer es una de las principales causas de muerte en el mundo. En 2008 la OMS informó de 12.4 millones de nuevos casos y 7.9 millones de defunciones (aproximadamente el 13\% de las defunciones mundiales). Aproximadamente el $72 \%$ de las defunciones por cáncer ocurridas en 2007 se registraron en países de ingresos bajos y medianos. Se prevé que el número de decesos anuales mundiales por cáncer seguirá aumentando y llegará a unos 12 millones en 2030 y el cáncer de mama es el más común entre las mujeres. Se estima que 636000 casos ocurrieron en países de primer mundo y 514000 en países en desarrollo durante el año 2002. En ese año hubo alrededor de 410 000 muertes por esta causa, en todo el mundo (Boyle \& Levin, 2008). En México, para el año 2003 se registraron 12,433 casos de cáncer de mama en mujeres de 40 a 59 años y en las de 70 y más años de edad, en un patrón bimodal relativamente consistente a lo largo de la década (Secretaría de Salud RHNM, 2003).

\section{Sufrimiento psicológico}

El diagnostico de cáncer suele ocasionar una serie de reacciones emocionales negativas y la expectativa de una vida en la cual el dolor se mantendrá presente. Entre las reacciones más frecuentes destacan las asociadas con sintomatología ansiosa y depresiva: disforia, anhedonia, trastornos del sueño, cambios en el apetito, fatiga, lentitud y/o agitación psicomotora, baja autoestima, culpa, dificultad para concentrarse, dificultad para tomar decisiones e ideación suicida (Mehnert \& Koch, 2008). En sentido psicológico el cáncer de mama en particular tiene una naturaleza insidiosa porque agrede a un órgano íntimamente asociado con la autoestima, la sexualidad y la femineidad (Biglia, et al., 2010; Lumbreras \& Blasco, 2008).

Aunque no todo el distrés emocional diagnosticado en pacientes de cáncer de mama puede calificarse como patológico, una alta proporción de las pacientes en estadio avanzado sufren de depresión grave (Thornton, Andersen \& Blakely, 2010). En el proceso de enfermedad destacan ocasiones críticas para el malestar psicológico entre las que figuran las siguientes.

El Diagnóstico suele valorarse como altamente traumático, en especial para mujeres menores de 45 años, pues, entre otras experiencias, genera sensación de poco control sobre los acontecimientos (Bell, Ziner \& Champion, 2009). El estrés suele ser especialmente elevado si la persona no consideraba la posibilidad del resultado diagnóstico e incluye sentimientos y actitudes de incredulidad, negación o desesperación, especialmente en condiciones culturales de relativa marginalidad (Ashida, Palmquist, Basen-Engquist, Singletary \& Koehly, 2009; Lam \& Fielding, 2003). Algunas pacientes refieren azoro, aturdimiento y alienación, desesperación y desmoralización a la que suele seguir una fase de disforia con sintomatología ansiosa o depresiva, problemas cognitivos (atención y concentración) y alteración de las actividades diarias (Davis, 2004; Munir, Burrows, Yarker, Kalawsky \& Bains 2010).

\section{Efectos colaterales del tratamiento médico}

Además del sufrimiento psicológico ocasionado por el diagnóstico, varios tratamientos contra el cáncer de mama como la radiación, quimioterapia o la cirugía ocasionan reacciones extremadamente molestas: náusea, vómito, ptialismo, mareos, aturdimiento, dificultad para digerir, cambios molestos en la temperatura de la piel y taquicardia, entre otros. Adicionalmente, la quimioterapia produce, pérdida del cabello, disminución del apetito, amoratamiento de uñas, diarrea, úlceras en la boca y conjuntivitis; altera el sentido del gusto y produce hiperosmia (Corner, 2009; Morrow, Lindke, Black 1991). Otros tratamientos generan adormecimiento en extremidades, mucositis, estreñimiento, cambios en la textura de la piel, alopecia púbica, sequedad vaginal, dolor durante el coito, anorgasmia y síntomas menopáusicos. La privación de estrógenos puede producir atrofia vaginal, estrechez de los tejidos vulvares y vaginales, infecciones urinarias, labilidad afectiva, cansancio e irritabilidad (Tamblay \& Bañados, 2004).

La radioterapia se ha asociado con leucemias secundarias, tumores sólidos, problemas de piel, cefalea, diarrea, somnolencia, fiebre y pérdida del apetito. Otra afección frecuente es la acumulación de linfa que provoca aumento progresivo del volumen del brazo con posible infección y síntomas como dolor, pirosis y enrojecimiento (Álvarez, 2003). A menudo, las pacientes se muestran temerosas de someterse a un tratamiento "invisible" que les obliga a permanecer inmóviles, en silencio y solas debajo de una gran máquina; otros prejuicios incluyen el que van a resultar quemadas, desfiguradas $o$ a perder funciones fisiológicas esenciales (Halkett, Kristjanson \& Lobb, 2008; Michielutte, Dignan \& Smith, 1999).

La mastectomía suele producir sentimientos de pérdida, mutilación, desvalorización de la imagen corporal. La amenaza a la femineidad suele ser más intensa en mujeres jóvenes cuyo atractivo y fertilidad está en auge, especialmente en solteras o sin pareja (Avis, Crawford \& Manuel, 2004; Fobair, et al., 2006; 
Sammarco, 2009). Aún cuando los tratamientos resultan exitosos en el intervalo libre de enfermedad las pacientes suelen sentirse vulnerables y desprotegidas ante un futuro incierto por el miedo a una recaída, temor que se incrementa en las revisiones y controles rutinarios. Se enfrentan al regreso al trabajo y a responsabilidades domésticas, con mucha menor energía, motivaciones y valores. Aquí la ayuda psicológica se orienta a reducir las secuelas subjetivas de la enfermedad y mejorar la readaptación a la vida cotidiana (Bellver, 2007).

El diagnóstico de metástasis o recaída demanda ajuste a la nueva información, que suele acompañarse de sentimientos de fracaso por la elección terapéutica, culpar al médico o reacciones que niegan la gravedad de la recurrencia; puede haber pérdida de la esperanza al creer que la recaída significa muerte inminente y una actitud de total abandono y resignación. También suelen evitarse las citas médicas o demorar la verificación de nuevas lesiones, seguidas por sintomatología depresiva, sensación de indefensión, pérdida de control, enojo, y preocupación por el futuro (Davis, 2004; Karademas, Argyropoulou \& Karvelis, 2007).

\section{Intervención Cognitivo-Conductual}

En general, la terapia cognitivo-conductual busca modificar las cogniciones y conductas que complican los problemas de salud a través de técnicas basadas en la investigación científica (Trull \& Phares, 2003). Desde sus proponentes clásicos esta terapia busca corregir patrones de pensamiento y creencias irracionales (Beck, 1976; Ellis, 1980; Goldfried \& Goldfried, 1987; Meichenbaum, 1977); enseñar técnicas de comunicación eficaz, reducir los efectos del estrés con afrontamiento eficaz y a autorregular reacciones emocionales negativas que añaden sufrimiento (Moorey \& Greer, 1989). Otras técnicas seminales persisten hasta la actualidad con pocas modificaciones e incluyen el establecimiento de destrezas a usarse en otras situaciones estresoras; destacan el entrenamiento en manejo de ansiedad (Suinn \& Richardson, 1971), la inoculación de estrés (Meichenbaum \& Cameron, 1983) y otras técnicas de autocontrol (Carrobles, 1985).

Se han usado intervenciones cognitivo conductuales sobre componentes psicológicos de problemas de salud, incluyendo el distrés relacionado con el cáncer y sus tratamientos (Hersch, Juraskova, Price \& Mullan, 2009); generalmente éstas buscan: 1. promover un ambiente de apoyo, 2. facilitar el manejo del duelo por las múltiples perdidas, 3. incrementar los recursos de afrontamiento y las habilidades de solución de problemas, 4. promover la percepción de dominio y autocontrol y 5 . anticipar las prioridades del futuro (Seitz, Besier \& Goldbeck, 2009).

La psico-educación ayuda a las pacientes a afrontar la ausencia de información vital para la toma de decisiones y comprensión, la aceptación del tratamiento médico y las preocupaciones interpersonales y existenciales (Phillips et al., 2008). Se proporciona información sobre la enfermedad, su tratamiento y el papel de las variables psicológicas en el afrontamiento, reducción de la ansiedad, la satisfacción del paciente con la atención recibida y adhesión a las indicaciones médicas (Zimmermann, Heinrichs \& Baucom, 2007).

\section{Calidad de Vida}

La Calidad de Vida entraña la percepción del individuo de su posición en la vida en el contexto de su cultura y sistema de valores, en relación con sus metas, expectativas, estándares e intereses (Brähler, Mühlan, Albani \& Schmidt, 2007; Harper \& Power, 1998; Ohaeri, Olusina \& Al-Abassi, 2006). En psicología incluye la funcionalidad y el bienestar cuando la salud es una variable que afecta componentes vitales como trabajo, ocio, autonomía, relaciones sociales, etc. (Howren, Christensen, Karnell \& Funk, 2010). La evaluación de la CV por el médico correlaciona bajo con la percibida por el paciente en casos de cáncer ya que, en general, los médicos no determinan con precisión la forma en que se sienten sus pacientes (Aa-Petersen, Pedersen, \& Groenvold, 2007; Velikova, et al., 2008). La CV en atención a la salud es relevante para planificar la atención con base en las necesidades de una población, evaluar resultados en ensayos clínicos y estudios sobre servicios de salud y tomar mejores decisiones sobre distribución de recursos (Koopmanschap, van Exel, van den Berg \& Brouwer, 2008; Pyne et al., 2008).

La literatura de investigación sobre intervenciones cognitivo conductuales en Calidad de Vida de pacientes con cáncer de mama en condiciones de relativa marginalidad social son muy escasas. Algunas intervenciones psicosociales sugieren un efecto positivo del apoyo social, el cambio de cogniciones y conductas y la información de apoyo (Galván, Buki, \& Garcés, 2009; Matthews \& Cook, 2009). Otros estudios, con participantes socio-demográficamente "normales" buscan limitar el deterioro por dolor físico, mejorar actividades cotidianas, tolerar efectos colaterales del tratamiento, implantar conductas de cuidado de su salud, reducir la ansiedad anticipatoria de recaídas y manejo de la pérdida del apoyo social 
(Costanzo, et al., 2007).

Estos estudios, sin embargo, rara vez atienden variables asociadas con déficits económicos, educativos y culturales tan frecuentes en países en transición económica. Dichas variables pueden ser definitorias tanto de la calidad de vida como de una atención exitosa y, por tanto, de mejores probabilidades de recuperación (Flórez, et al., 2009; Sperry, 2010; Zimmermann, Heinrichs \& Baucom, 2007).

En México un estudio sometió a prueba un sistema de auto-registro conductual para sondear los efectos de una intervención conductual como apoyo al tratamiento y reducción del estrés en pacientes de baja escolaridad con cáncer mamario. A guisa de prueba piloto, el estudio incluyó las primeras seis pacientes que completaron el protocolo de un grupo de 87, con máximo de primaria inconclusa. Los resultados apuntan hacia un sistema confiable, económico y práctico de registro y mejoría notoria en la mayoría de las categorías conductuales en las participantes (Sánchez-Sosa \& Alvarado, 2008).

Pocos trabajos publicados en Latinoamérica evalúan intervenciones psicológicas en pacientes con cáncer y prácticamente ninguno detalla procedimientos terapéuticos. Un estudio buscó disminuir la intensidad del dolor y de la sintomatología depresiva y ansiosa con terapia cognitivo-conductual en siete pacientes. Los resultados mostraron reducción estadísticamente significativa en la intensidad de la sintomatología aunque sólo dos pacientes mostraron decremento significativo en la intensidad del dolor percibido al final del tratamiento (Acosta, 2003).

En el contexto de que un elemento medular para reducir el deterioro y el sufrimiento de las pacientes lo constituye el propio comportamiento humano (Sánchez-Sosa, 2002); que el cáncer de mama va en aumento en México y que genera sufrimiento intenso y prolongado, psicológico y físico, es importante mejorar la calidad de vida, la funcionalidad y el bienestar de las pacientes. Así, el propósito del presente estudio fue examinar el efecto de un tratamiento sobre aspectos emocionales, cognitivos e instrumentales asociados a la calidad de vida en pacientes de bajos recursos y escolaridad.

\section{Método}

\section{Participantes}

Participaron pacientes del Instituto Nacional de Cancerología de la ciudad de México, de sexo femenino con diagnóstico confirmado de cáncer de mama. Algunas participantes potenciales se excluyeron del es- tudio si presentaban un cuadro psiquiátrico o retraso mental, si se encontraban en fase terminal y cuidados paliativos, o si participaban en otro protocolo de investigación. Todas las participantes o sus cuidadores primarios tenían un ingreso mensual que los situaba en el promedio de salario mínimo (aproximadamente cuatro dólares diarios) o ligeramente por arriba de éste. La mayoría vivían en pareja (45\%) y eran amas de casa (47\%). Tenían educación básica (hasta nueve años) el $52 \%$ y eran católicas el $87 \%$. El grupo final quedó conformado por 60 pacientes con edades entre los 31 y 67 años. Su evolución de la enfermedad era de 3 meses a 7 años desde el diagnóstico. A las pacientes se las reclutó en la sala de espera del pabellón de tumores mamarios del hospital o en los consultorios médicos del mismo.

\section{Medición}

Los principales datos se recolectaron mediante el Inventario de Calidad de Vida y Salud o "InCaViSa" (Riveros, Sánchez-Sosa \& Del Águila, 2009), compuesto de 53 reactivos tipo Likert en doce áreas de Calidad de Vida: Preocupaciones, Desempeño físico, Aislamiento, Percepción corporal, Funciones cognitivas, Actitud ante el tratamiento, Tiempo libre, Vida cotidiana, Familia, Redes sociales, Dependencia médica, y Relación con el médico. El inventario también incluye una sección de cuatro reactivos sobre información o comentarios generales y sobre situaciones transitorias que pudieran afectar los resultados de la escala (disgustos, problemas de salud, muerte de alguien cercano, separación, etc.); así como comentarios generales sobre el padecimiento o el instrumento y una sección de datos socioeconómicos.

Los reactivos se califican en escala de opción forzosa con tres componentes cuantificables: a) porcentual que va de $0 \%$ a $100 \%$; b) semántico que va de "nunca" a "siempre"; y c) visual sobre una recta continua con extremos de $0 \%$ (nunca) hasta $100 \%$ (siempre). A los reactivos se les asigna un valor de 0 a 5 puntos. A continuación se enlistan las áreas del instrumento con un espécimen de reactivo.

- Preocupaciones: "Me preocupa que alguien se sienta incómodo(a) cuando está conmigo, por mi enfermedad".

- Desempeño Físico: "Puedo cargar las bolsas del mandado sin hacer grandes esfuerzos".

- Aislamiento: "Me parece que desde que enfermé no confían en mí como antes".

- Percepción Corporal: "Me da pena mi cuerpo".

- Funciones Cognitivas: "Se me olvida en dónde 
puse las cosas".

- Actitud ante el Tratamiento "Los efectos de las medicinas son peores que la enfermedad".

- Tiempo Libre: "Desde que enfermé dejé de pasar el rato con mis amigos".

- Vida Cotidiana: "Ahora que estoy enfermo(a) la vida me parece aburrida".

- Familia: "Mi familia cuida mis sentimientos".

- Redes Sociales "Cuando las cosas me salen mal hay alguien que me puede ayudar".

- Dependencia Médica: "No me importa qué tengo, sólo quiero que el médico me cure".

- Relación con el médico: "Me gusta hablar con el médico sobre mi enfermedad".

Diversos estudios y análisis realizados durante la construcción del instrumento arrojaron medidas altas y confiables en varias dimensiones. Se aplicó el inventario a tres grupos: un grupo de pacientes con padecimientos crónicos $(n=172)$, uno con padecimientos agudos $(n=129)$ y un control de sanos $(n=114)$, para una $\mathrm{N}$ total de 435 participantes. La consistencia interna arrojó valores Alfa de Cronbach que oscilaron entre .9337 para el área de Vida Cotidiana y .6831 para Actitud ante el tratamiento. Un análisis factorial con rotación varimax sobre la estructura de la escala culminó en cada área compuesta por cuatro reactivos y el total de doce áreas explicó el $68 \%$ de la varianza.

El instrumento ha mostrado estabilidad temporal y validez concurrente con el OMS-QoL Bref en su versión adaptada para México (Sánchez-Sosa \& González-Celis, 2003). También ha resultado útil para evaluar el efecto de intervenciones para mejorar la calidad de vida en comparaciones pre-post tratamiento; en seguimientos muestra buena estabilidad y posibilidad de reconocer el efecto de situaciones transitorias (Riveros, Castro \& Lara-Tapia, 2009)

Adicionalmente, cada sesión se guiaba por una forma denominada Bitácora Clínica en que se anotaban procedimientos, registros, intervenciones y comentarios del terapeuta o la paciente.

\section{Recolección de datos}

Todas las entrevistas, recolección de datos y procedimientos de intervención estuvieron a cargo de siete estudiantes avanzados de la licenciatura en psicología de la Universidad Nacional Autónoma de México. A los estudiantes se les seleccionó a partir de sus calificaciones en cursos avanzados específicos en terapia cognitivo conductual y recibieron durante otros dos semestres entrenamiento adicional en intervenciones con pacientes con cáncer de mama. Su trabajo como terapeutas se dio bajo la supervisión de un psicólogo que cursaba un posgrado en Medicina Conductual y por el investigador principal del programa.

\section{Procedimientos generales}

Inicialmente se describía a las pacientes el programa con énfasis en el papel del comportamiento en el tratamiento del cáncer y en los beneficios potenciales de la participación en el programa. Se les presentaba y explicaba una Carta de Consentimiento Informado con las características del estudio, sus posibles beneficios, responsabilidades adquiridas, garantía de anonimato, confidencialidad y la libre opción de dejar el estudio en cualquier momento sin consecuencias institucionales de ninguna índole. Finalmente se les ofrecía asesoría adicional después de completar los protocolos del proyecto si así lo requería. Una vez reclutadas se las asignaba a un calendario de sesiones en consultorios del hospital. Ocasionalmente las sesiones se realizaban en la casa del paciente en caso de recuperación post-quirúrgica o por imposibilidad para transportarse al hospital.

\section{Procedimientos Clínicos}

La intervención tuvo modalidad individual, de corta duración, activa, focalizada, y directiva en consultas semanales de una hora en un promedio de 16 sesiones. En la primera sesión se llenaba el formato de entrevista y datos personales y socio-demográficos de la paciente.

\section{Psico-educación}

Se la exponía al contenido de un texto aproximadamente seis cuartillas sobre el cáncer de mama con énfasis en sus aspectos psicológicos en un castellano muy sencillo. Si la paciente no sabía leer se leían, explicaban y ejemplificaban los contenidos del texto en términos sencillos y se la invitaba a hacer preguntas. Se explicaban los objetivos terapéuticos: a) establecimiento de conductas de apoyo al tratamiento médico, b) modificación de pensamientos generadores de sufrimiento o incompatibles con su tratamiento, c) adquisición de habilidades para mitigar reacciones emocionales negativas. También se les explicaba que cualquier futura pregunta y dudas serían bienvenidas en cualquier momento durante el tratamiento.

\section{Componentes Instrumentales}

El establecimiento de habilidades y conductas especificas siguieron la línea de solución de problemas de Safren, et al. (2001); Nezu, Palmatier \& Nezu (2004) y 
D'Zurilla, Nezu \& Maydieu-Olivares (2004). Los terapeutas revisaban en detalle, con la paciente una lista de conductas de adhesión terapéutica y posibles problemas y soluciones titulada "Pasos Vitales" (Safren, Otto \& Worth, 1999). Para cada problema identificado se analizaba con la paciente la viabilidad de las soluciones posibles y se la motivaba para ponerlos en práctica. Las principales acciones de adhesión incluían: presentarse a consulta, preguntar al personal médico, obtener y administrar medicamentos, tolerancia a los efectos colaterales, higiene personal y preparación para su atención médica.

\section{Componentes Cognitivos}

Los procedimientos para detectar y modificar creencias distorsionadas o disfuncionales se basaron en las técnicas de cambio de valoraciones de la paciente (Brewin, 1996), y en los de la psicoterapia funcional analítica (Kanter, Landes, Busch, Rusch, Brown \& Baruch, 2006; Kohlenberg, Kanter, Bolling, Parker \& Tsai, 2002; Vandenberghe, 2008). Así, cuando una paciente verbalizaba una idea disfuncional el terapeuta cuestionaba su plausibilidad directamente o conducía a un ejercicio de preguntas y respuestas, guiándola a desarrollar una (o ambas) de dos conclusiones: qué tan irracional o irreal era la creencia, y cómo interfería con su bienestar y tratamiento médico. Si la paciente mostraba cambio positivo en las verbalizaciones correspondientes el terapeuta le hacía un reconocimiento verbal explicando cómo la idea nueva mejoraría su bienestar y apoyaría su tratamiento.

\section{Componentes Emocionales}

Los principales procedimientos para disminuir reacciones psico-fisiológicas de ansiedad incluían el entrenamiento en respiración diafragmática profunda y relajación muscular progresiva profunda (Fahrion \& Norris, 1990; Lehrer, 1996). Después de explicar a la paciente la relación entre ansiedad y tensión muscular en términos simples, el terapeuta la instaba a sentarse cómodamente, aflojar su ropa e imitar la respiración diafragmática modelada por el terapeuta. Se incluía respirar profundamente y monitorear el componente diafragmático colocando su mano sobre el abdomen. El terapeuta usaba frases pausadas para marcar un ritmo respiratorio profundo. Se inducían respiraciones profundas, lenta (unos 8 segundos del inicio de la inhalación al final de la exhalación), desde el abdomen y cómoda, y se reforzaba verbalmente a la paciente por su progreso. Una vez que el terapeuta describía y ejemplificaba el contraste entre tensiónrelajación se pedía a las pacientes que tensaran por unos segundos y después relajaran grupos específicos de músculos. Esta alternancia tensión-relajación usó la secuencia: frente, mejillas, labios, mandíbula, cuello, hombros, brazos, manos, abdomen, trasero, piernas, tobillos y pies.

Al final de cada sesión el terapeuta asignaba a la paciente tareas para realizarse en casa con instrucciones adicionales si había colaboración de cuidadores primarios. En estos casos, los familiares hacían registros de confiabilidad llenando los formatos sin comunicarse con la paciente. Un análisis de $10 \%$ de los registros, seleccionados aleatoriamente arrojó acuerdo independiente superior a $80 \%$. A fin de reducir posibles sesgos, el entrenamiento de terapeutas incluía instrucciones específicas para apegarse a los protocolos de intervención y evitar cualquier interacción verbal con la paciente o sus familiares, que pudiera inducir cambios espuriamente.

\section{Resultados}

A fin de valorar la posible normalidad de distribución de las dimensiones del estudio, se computó la prueba de Kolmogorov-Smirnov, misma que reveló que, de las 48 distribuciones sometidas a prueba, 18 no se distribuían de manera normal. En tal virtud, las diferencias entre las cuatro mediciones (pre-test, post-test y seguimientos uno y dos) se analizaron con la prueba de Friedman por tratarse, además, de escalas con estructura ordinal. Los valores de chi-cuadrada oscilaron entre 10.76 y 59.24 con probabilidades asociadas entre .013 y .001 . Los datos de las áreas negativas se redujeron consistentemente de la pre prueba al segundo seguimiento. Excepto en el caso de "relación con el médico" (X2=7.6, $p=0.054)$ la mejoría reflejó cambios consistentes entre las medias.

La magnitud del cambio se calculó mediante las pruebas $t$ y Wilcoxon. La tabla 1 muestra las áreas con la diferencia entre las medias del pre al postratamiento, y los valores y probabilidades asociadas de ambos estadísticos. Los valores de las pruebas se replican entre sí, con los mayores beneficios en las áreas de preocupaciones, uso del tiempo libre, y vida cotidiana. Sólo relación con el médico no mostró mejoría significativa, aunque el signo indica que la relación mejoró.

La tabla 2 muestra la distribución de pacientes por tipo y tamaño del cambio. De izquierda a derecha las columnas representan: las áreas de Calidad de Vida, las pacientes cuya mejora (+) fue mayor a $75 \%$ seguidas, sucesivamente de las mejoraron más de $50 \%$, más de $25 \%$ y de 0 a $24 \%$; el total de pacientes que 
mejoraron $y$, en las últimas dos columnas las que mostraron algún empeoramiento, de $1 \%$ a $25 \%$ y de $26 \%$ a $50 \%$, (-) y algunos casos que mostraron mayor empeoramiento. Excepto en las áreas de desempeño físico, dependencia médica y en un caso de familia, la cantidad de mejoría en la gran mayoría de las pacientes fue mayor a $25 \%$.

\section{Discusión}

El propósito principal del presente estudio consistió en examinar los efectos de una intervención Cognitivo Conductual sobre la Calidad de Vida de pacientes con diagnóstico confirmado de cáncer de mama.

Los resultados señalan que la gran mayoría de las pacientes mostraron cambios positivos estadística y clínicamente significativos. Como suele ocurrir en los estudios de carácter clínico hubo pacientes cuya mejoría fue muy importante, la mayoría mostró beneficios medianos $y$, en algunas dimensiones, el cambio fue negativo para un número pequeño de participantes.

De las doce áreas de las que se compone el InCaVisa la mejoría más importante se dio en Vida Cotidiana, Tiempo Libre, Preocupaciones, Percepción Corporal y Aislamiento. Es probable que esta mejoría haya surgido en buena parte del hecho de que la intervención psicológica busca precisamente dotar a la paciente de un repertorio cognitivo conductual, incrementado y funcional, que le permita afrontar de mejor forma las situaciones que se le presentan en función del padecimiento y de las dificultades típi-

Tabla 1. Prueba t de Muestras Relacionadas y análisis de Wilcoxon (Pre-Seg.2).

\begin{tabular}{|c|c|c|c|c|c|}
\hline & Dif.Medias & $\mathrm{t}$ & $\mathrm{p}$ & z & $\mathrm{p}$ \\
\hline Preocupaciones & 4.967 & 7.434 & $<.001$ & -5.679 & $<.001$ \\
\hline Desempeño Físico & -2.050 & -3.292 & .002 & -3.254 & $<.001$ \\
\hline Aislamiento & 2.633 & 4.326 & $<.001$ & -4.629 & $<.001$ \\
\hline Percepción Corporal & 2.450 & 3.793 & $<.001$ & -3.643 & $<.001$ \\
\hline Funciones Cognitivas & 2.683 & 4.102 & $<.001$ & -3.753 & $<.001$ \\
\hline Actitud al Tratamiento & 2.500 & 4.788 & $<.001$ & -4.425 & $<.001$ \\
\hline Tiempo Libre & 3.650 & 5.676 & $<.001$ & -4.773 & $<.001$ \\
\hline Vida Cotidiana & 3.517 & 5.330 & $<.001$ & -4.826 & $<.001$ \\
\hline Familia & -.950 & -1.926 & .059 & -1.965 & .049 \\
\hline Redes Sociales & -2.150 & -3.882 & $<.001$ & -3.574 & $<.001$ \\
\hline Dependencia Médica & 2.400 & 2.967 & .004 & -2.905 & .004 \\
\hline Relación con Médico & -.433 & -.779 & .439 & -.661 & .508 \\
\hline
\end{tabular}


Tabla 2. Frecuencia de Pacientes por Porcentaje de cambio. Mejor+. Peor- $(\mathrm{N}=60)$

\begin{tabular}{|c|c|c|c|c|c|c|c|}
\hline ÁREA & $>75 \%+$ & $50-74 \%+$ & $25-49 \%+$ & $0-24 \%+$ & Total & $1-25 \%-$ & $26-50 \%$ \\
\hline Preocupaciones & $2 \quad 9$ & 19 & 21 & 51 & 9 & 0 & \\
\hline Tiempo Libre & 2 & 5 & 17 & 27 & 51 & 8 & 1 \\
\hline Vida-Cotidiana & 3 & 4 & 12 & 32 & 51 & 8 & 1 \\
\hline Actitud-Tratamiento & 2 & 2 & 11 & 36 & 51 & 9 & 0 \\
\hline Aislamiento & 3 & 1 & 9 & 37 & 50 & 9 & 1 \\
\hline Percepción Corporal & 3 & 2 & 8 & 33 & 46 & 12 & 2 \\
\hline Dependencia Médica & 0 & 10 & 12 & 22 & 44 & 9 & $4 / 3^{*}$ \\
\hline Funciones Cognitivas & 2 & 3 & 15 & 24 & 44 & 13 & 3 \\
\hline Familia & 0 & 1 & 1 & 37 & 39 & 16 & $4 / 1^{*}$ \\
\hline Redes Sociales & 0 & 0 & 3 & 35 & 38 & 20 & 2 \\
\hline Relación-Médico & 0 & 0 & 8 & 24 & 32 & 19 & 9 \\
\hline Desempeño Físico & 0 & 1 & 3 & 16 & 20 & 26 & $11 / 3^{*}$ \\
\hline TOTAL & 17 & 38 & 118 & 344 & 517 & 158 & 45 \\
\hline
\end{tabular}

* $50 \%$ de empeoramiento o más.

cas de un nivel socioeconómico relativamente bajo (Sanchez Sosa \& Alvarado, 2008). En el caso de las pacientes con cáncer de mama se trata de mantener un funcionamiento con las menores contrariedades posibles, sin mucho tiempo libre desperdiciado que, pudiera genera demasiada atención a síntomas y molestias del tratamiento mismo. También se busca que, a pesar de los cambios emocionales y cognitivos ocasionados por el diagnóstico y el tratamiento las rutinas cotidianas se perciban como reflejo de estabilidad y relativa satisfacción.

El uso satisfactorio del Tiempo Libre también mostró mejoría notoria. Es probable que la percepción de la paciente en relación a que las dificultades que enfrenta no obstaculicen seriamente sus actividades recreativas. Las pacientes aprendían que mientras más llevaran una vida normal, menos daños se notarían debido a la enfermedad. Aprendían a conservar actividades como reuniones fami-liares o con amistades, visitas a cines, teatros, instalaciones de actividades deportivas, restaurantes etc. Al respecto de sus preocupaciones aprendían que el estrés generado en una preocupación podría llegar a ser perjudicial a la salud y a aplicar las estrategias aprendidas del tratamiento.

Entre las preocupaciones mejoradas estuvieron la incomodidad por problemas económicos, un exagerado acogimiento por parte de cuidadores primarios, que dicho apoyo se convirtiera en una carga, de desgaste físico o emocional o incomodidad por estar enferma. La percepción corporal también mostró mejorías importantes probablemente ligada a la percepción del estado físico y la satisfacción al respecto de él.

Hubo áreas que mostraron ganancias más modestas tales como las relativas a la Relación con el Médi- 
co, Redes Sociales, Familia y Desempeño Físico. En el caso de la relación con el cuerpo médico es probable que se trate no tanto de déficits serios en habilidades interpersonales de la paciente sino formas peculiares de interacción (quizá conformadas institucionalmente) con sus interlocutores. Las pacientes aprendieron a afrontar situaciones difíciles con su especialista, con el encargado de área, con las enfermeras etc. En efecto, las notas en las bitácoras clínicas sugieren que dichas relaciones sí mejoraron con otros miembros del personal aunque un tanto menos con el médico. Es probable que sea precisamente el cuerpo médico el que carezca de entrenamiento específico (o motivación) en esta área. Este es un hallazgo importante en relación a que los instrumentos existentes sobre Calidad de Vida no lo incluyen.

Las Redes Sociales mostraron una ganancia modesta probablemente debido a que no es fácil para algunas pacientes salir y convivir con sus amistades por cambios en el aspecto personal. La mayoría reportaba una preferencia por pasar la situación de enfermedad con su familia inmediata por su cercanía natural. Con amistades preferían mantener otro tipo de contactos tales como telefónico o con visitas sólo ocasionales. Algunas pacientes prefirieron no hacerle saber a toda su gente que estaban enfermas ya que deseaban dar "la sorpresa de éxito en el tratamiento".

En el caso de la familia, se nota un mantenimiento positivo del apoyo familiar, aunque con poca ganancia probablemente debido a un efecto de "techo" dado que en la gran mayoría de los casos había buen apoyo por parte de la familia y de los cuidadores primarios.

La restructuración cognitiva puede haber generado buena parte de las ganancias en la forma de afrontar los problemas de las pacientes. Según las bitácoras clínicas, un comentario inicial, frecuente de las pacientes refería pensamientos irracionales tales como que, el hecho de estar enfermas se debía a estar pagando errores cometidos en el pasado, por castigo divino, por haber sido malas personas, por ser iracundas, etc. Un componente central de la intervención consistió precisamente en reemplazar estos pensamientos por otros más positivos para sobrellevar su situación e involucrarse en su auto-cuidado. Naturalmente, las notas clínicas de las bitácoras no fueron objeto de análisis formal pues su categorización hubiera añadido muy poca información a los principales hallagos del estudio y su propósito era cotejar cambios aparentemente menores pero clínicamente relevantes (Prentice \& Miller, 1992).

El área de desempeño físico mostró poca mejoría muy probablemente por los efectos colaterales ya mencionados. Puede haber sido difícil mantener una actividad física ya que, aún ante mejoría, ésta se veía mermada por quimioterapia, radiación o tratamientos paliativos. Cabe destacar, de acuerdo con las bitácoras clínicas, la gran mayoría de las pacientes en estos casos mantuvo una actitud positiva a pesar de las circunstancias.

En cuanto a la regulación emocional, la relajación muscular progresiva profunda puede haber sido uno de los componentes más provechosos, es posible que las haya dotado de mayor estabilidad emocional al afrontar situaciones estresantes. Entre otras fuentes de estrés intenso o sostenido las pacientes destacaron: la náusea provocada por la quimioterapia, el dolor e incomodidad de los estados post-quirúrgicos, la adaptación al ambiente hospitalario con su ritmo acelerado, situaciones médicas o familiares estresantes, el tiempo en el transporte público o privado al hospital, etc. Las pacientes por lo general referían sentirse más tranquilas, incluso físicamente, para continuar su vida con mayor calma y ánimo.

Los hallazgos del presente estudio amplían los de la literatura internacional, en el sentido de que las intervenciones Cognitivo-Conductuales resultan eficaces para mejorar diversos aspectos psicológicos que a su vez resultan componentes de la dimensión denominada Calidad de Vida en pacientes con baja escolaridad y restricciones económicas (Lam \& Fielding, 2003; Zimmermann, Heinrichs, \& Baucom, 2007). Por ejemplo, una proporción importante de participantes mostraba analfabetismo funcional: si bien leían correctamente en voz alta un texto, su comprensión de la lectura era mínima (Parra, Mazón, Rojas-Drummond \& Espinosa-Vélez, 2005).

Será importante, para estudios futuros ampliar el número de participantes para mejorar la confiabilidad de los resultados y disponer de un estudio más sólido en sentido metodológico. También sería conveniente explorar la correlación de los resultados del instrumento y el tipo de tratamiento médico-oncológico que lleva la paciente. Sería relevante estudiar las estrategias de afrontamiento de las pacientes. También convendría añadir a la entrevista inicial preguntas surgidas de la presente experiencia a fin de establecer módulos de intervención a cada necesidad primaria de la paciente. Se podría ampliar la intervención a familiares, amigos y cuidadores primarios, incluir sesiones médico-psicológicas con el personal a cargo y actualizar periódicamente la información y avances de la paciente.

Para aquellos casos de las pacientes que no consiguieron mayor beneficio sería recomendable iden- 
tificar componentes donde la paciente no responde adecuadamente y diseñar intervenciones más específicas. En sentido clínico convendría instrumentar un seguimiento telefónico más específico y prolongado, mayor contacto con cuidadores primarios y familiares y mejorar la comunicación con el cuerpo médico y administrativo que instrumenta el tratamiento de la paciente.

\section{Referencias}

Aa-Petersen, M., Pedersen, L. \& Groenvold, M. (2007). Does the agreement of patient and physician assessments of health related quality of life in palliative care depend on patient characteristics?. Palliative Medicine, 21(4), 289-294.

Acosta, C. (2003). Tratamiento cognitivo conductual en el manejo del dolor por cáncer. Congreso de la Asociación Mexicana para el Estudio del Dolor. Oaxaca. México.

Álvarez, G. A. (2003). Propuesta de un manual para padres de niños con cáncer. Tesis de licenciatura. Universidad Intercontinental.

Ashida, S., Palmquist, A. E., Basen-Engquist, K., Singletary, S. E. \& Koehly, L. M. (2009). Changes in female support network systems and adaptation after breast cancer diagnosis: Differences between older and younger patients. The Gerontologist, 49(4), 549-559.

Avis, N. E., Crawford, S \& Manuel, J. (2004). Psychosocial problems among younger women with breast cancer. Psycho-Oncology, 13(5), 295-308.

Beck, A.T. (1976). Cognitive Therapy and the Emotional Disorders. New York: International University Press.

Bell, C. J., Ziner, K. W.\& Champion, V. L. (2009). Death worries and quality of life in younger breast cancer survivors. Western Journal of Nursing Research, 31(8), 1076-1077.

Bellver, A. (2007). Eficacia de la terapia grupal en la calidad de vida y el estado emocional en mujeres con cáncer de mama. Psicooncología, 4(1), 133-142.

Biglia, N., Moggio, G., Peano, E., Sgandurra, P., Ponzone, R., Nappi, R. \& Sismondi, P. (2010). Effects of surgical and adjuvant therapies for breast cancer on sexuality, cognitive functions, and body weight. Journal of Sexual Medicine, 7(5), 1891-1900.

Boyle, P. \& Levin, B. (2008). World Cancer Report. Lyon: IARC-WHO.

Brähler, E., Mühlan, H., Albani, C. \& Schmidt, S. (2007). Testing and standardization of the Ger- man version of the EUROHIS-QOL and WHO-5 quality-of life-indices. Diagnostica, 53(2), 83-96. Brewin, C.R. (1996). Theoretical foundations of cognitive-behavior therapy for anxiety and depression. Annual Review of Psychology, 47(1), 33-57.

Carrobles, J. A. (1985). El modelo conductual o del aprendizaje social: Enfoques y aplicaciones. En J. A. Carrobles (Ed.), Análisis y Modificación de conducta II (pp.77-108). Madrid: UNED.

Corner, J. (2009). Harnessing technology to improve support for people with cancer. Journal of Research in Nursing, 14(1), 9-11.

Costanzo, E. S., Lutgendorf, S.K., Mattes, M.L., Trehan, S., Robinson, C.B., Tewfik, F. \& Roman, S.L. (2007). Adjusting to life after treatment: distress and quality of life following treatment for breast cancer. British Journal of Cancer, 97(12), 1625-31.

Davis, C. (2004) Psychosocial Needs of Women with Breast Cancer: How Can Social Workers Make a Difference?. Health \& Social Work, 29(4), 330334.

D'Zurilla, T.J., Nezu, A.M. \& Maydeu-Olivares, A. (2004). Social Problem Solving: Theory and Assessment. In: E. C. Chang, T. J. D'Zurilla \& L. J. Sanna (Eds). Social Problem Solving: Theory, Research, and Training (pp. 11- 27). Washington, DC: American Psychological Association.

Ellis, A. (1980). Rational emotive therapy and cognitive behavior therapy: Similarities and differences. Cognitive Therapy \& Research, 4(3), 325-340.

Fahrion, S.L. \& Norris, P.A. (1990). Self-regulation of anxiety. Bulletin of the Menninger Clinic, 54(2), 217-231.

Flórez, K. R., Aguirre, A. N., Viladrich, A., Céspedes, A., De La Cruz, A. A. \& Abraído-Lanza, A. F. (2009). Fatalism or destiny? A qualitative study and interpretative framework on Dominican women's breast cancer beliefs. Journal of Immigrant and Minority Health, 11(4), 291-301.

Fobair, P., Stewart, S. L., Chang, S., D'Onofrio, C., Banks, P. J. \& Bloom, J. R. (2006). Body image and sexual problems in young women with breast cancer. Psycho-Oncology, 15(7), 579-594.

Galván, N., Buki, L. P. \& Garcés, D. M. (2009). Suddenly, a carriage appears: Social support needs of Latina breast cancer survivors. Journal of Psychosocial Oncology, 27(3), 361-382.

Goldfried, M.R. \& Goldfried, A.P. (1987). Métodos de cambio cognitivo. En F.H. Kanfer \& A.P. Goldstein (Eds.), Cómo ayudar al cambio en psicotera- 
pia: Un manual de métodos (pp. 123-165). Bilbao: Desclée de Brouwer.

Halkett, G. K. B., Kristjanson, L. J. \& Lobb, E. A. (2008). 'If we get too close to your bones they'll go brittle': Women's initial fears about radiotherapy for early breast cancer. Psycho-Oncology, 17(9), 877-884.

Harper, A., \& Power, M. (1998). Development of the World Health Organization WHOQoL-Bref quality of life assessment. Psychological Medicine, 28(6) 551-558.

Hersch, J., Juraskova, I., Price, M. \& Mullan, B. (2009). Psychosocial interventions and quality of life in gynaecological cancer patients: A systematic review. Psycho-Oncology, 18(8), 795-810.

Howren, M. B., Christensen, A. J., Karnell, L. H. \& Funk, G. F. (2010). Health related quality of life in head and neck cancer survivors: Impact of pretreatment depressive symptoms. Health Psychology, 29(1), 2010, 65-71.

Kanter, J.W., Landes, S.J., Busch, A.M., Rusch, L.C., Brown, K.R. \& Baruch, D.E. (2006). The Effect of Contingent Reinforcement on Target Variables in Outpatient Psychotherapy for Depression: A Successful and Unsuccessful Case Using Functional Analytic Psychotherapy. Journal of Applied Behavior Analysis, 39(4), 463-467.

Karademas, E. C., Argyropoulou, K. \& Karvelis, S. (2007). Psychological symptoms of breast cancer survivors: A comparison with matched healthy controls and the association with cancer-related stress and coping. Journal of psychosocial Oncology, 25(3), 59-74.

Kohlenberg, R.J., Kanter, J.W., Bolling, M.Y., Parker, C.R. \& Tsai, M. (2002). Enhancing cognitive therapy for depression with functional analytic psychotherapy: Treatment guidelines and empirical findings. Cognitive and Behavioral Practice, 9(3), 213-229.

Koopmanschap, M. A., van Exel, J. N. A., van den Berg, B. \& Brouwer, W. B. F. (2008). An overview of methods and applications to value informal care in economic evaluations of healthcare. PharmacoEconomics, 26(4), 269-280.

Lam, W. T. \& Fielding, R. (2003). The evolving experience of illness for Chinese women with breast cancer: A qualitative study. Psycho-Oncology, 12(2), 127-140.

Lehrer, P.M. (1996). Varieties of relaxation methods and their unique effects. International Journal of Stress Management, 3(1), 1-15.

Lumbreras, S. G. \& Blasco, T. (2008). Características de la sexualidad en mujeres con cáncer de mama. Psicooncología, 5(1), 155-170.

Massie, M.C. \& Holland, J.C. (1990) Depression and the cancer patient. Journal of clinical Psychiatry, 51(1), 12-17.

Matthews, E. E. \& Cook, P. F. (2009). Relationships among optimism, well-being, self-transcendence, coping, and social support in women during treatment for breast cancer. Psycho-Oncology, 18(7), 716-726.

Mehnert, A. \& Koch, U. (2008). Psychological comorbidity and health-related quality of life and its association with awareness, utilization, and need for psychosocial support in a cancer register-based sample of long-term breast cancer survivors. Journal of Psychosomatic Research, 64(4), 383-391.

Meichenbaum, D.H. (1977). Cognitive behavior modification: An integrative approach. Nueva York: Plenum Press.

Meichenbaum, D.H. \& Cameron, R. (1983). Stress inoculation training: Toward a general paradigm for training coping skills. En D. Meichenbaum y M.E. Jarenko (Eds.) Stress Reduction and Prevention. New York: Plenum Press.

Michielutte, R., Dignan, M. B. \& Smith, B. L. (1999). Psychosocial factors associated with the use of breast cancer screening by women age 60 years or over. Health Education \& Behavior, 26(5), 625-647.

Moorey S, Greer S. (1989) Psychological Therapy for Patients with Cancer: A new Approach. Oxford: Heinemann Medical Books.

Morrow, G., Lindke, J. \& Black, P.M. (1991). Anticipatory nausea development in cancer patients: replications and extension of a learning model. British Journal of Psychology, 82(1), 61-72.

Munir, F., Burrows, J., Yarker, J., Kalawsky, K. \& Bains, M. (2010). Women's perceptions of chemotherapy-induced cognitive side affects on work ability: A focus group study. Journal of Clinical Nursing, 19(9-10), 1362-1370.

Nezu, C.M., Palmatier, A.D. \& Nezu, A.M. (2004) Problem-Solving Therapy for Caregivers. In E. C. Chang, T. J. D'Zurilla, L. J. Sanna \& J. Lawrence (Eds.) Social problem solving: Theory, Research, and Training (pp. 223- 238). Washington, DC: American Psychological Association.

Ohaeri, J. U., Olusina, A. K. \& Al-Abassi, A. M. (2006). Path Analytical Study of the Short Version of the WHO Quality of Life Instrument. Psychopathology, 39(5), 243-247. 
Parra, N., Mazón, C., Rojas-Drummond, S. \& EspinosaVélez, M. (2005). Efectos de un programa de fortalecimiento de habilidades de comprensión de textos en educandos de primaria. Revista Mexicana de Psicología, 22(1), 91-102.

Phillips, K. M., Antoni, M. H., Lechner, S. C., Blomberg, B. B., Llabre, M. M., Avisar, E., Glück, S., DerHagopian, R. \& Carver, C. S. (2008). Stress management intervention reduces serum cortisol and increases relaxation during treatment for nonmetastic breast cancer. Psychosomatic Medicine, 70(9), 1044-1049.

Prentice, D. A. \& Miller, D. T. (1992). When small effects are impressive. Psychological Bulletin, 112(1), 160-164.

Pyne, J. M., French, M., McCollister, K., Tripathi, S., Rapp, R. \& Booth, B. (2008). Preferenceweighted health-related quality of life measures and substance use disorder severity. Addiction, 103(8), 1320-1329.

Riveros, A., Castro, C. G. \& Lara-Tapia, H. (2009). Características de la calidad de vida en enfermos crónicos y agudos. Revista Latinoamericana de Psicología, 41(2), 291-304.

Riveros, A., Sánchez-Sosa, J.J. \& Del Águila M. (2009). Inventario de Calidad de Vida y Salud (InCaViSa). México, Manual Moderno.

Safren, S.A., Otto, M.W. \& Worth, J.L. (1999). LifeSteps: Applying Cognitive Behavioral Therapy to HIV Medication Adherence. Cognitive and Behavioral Practice, 6(4), 332-341.

Safren, S.A., Otto, M.W., Worth, J.L., Salomon, E., Johnson, W., Mayer, K. \& Boswell, S. (2001). Two strategies to increase adherence to HIV antiretroviral medication: Life-steps and medication monitoring. Behaviour Research and Therapy, 39(10), 1151-1162.

Sammarco, A. (2009). Quality of life of breast cancer survivors: A comparative study of age cohorts. Cancer Nursing, 32(5), 347-356.

Sánchez-Sosa, J.J. (2002). Treatment adherence: The role of behavioral mechanisms and some implications for health care interventions. Revista Mexicana de Psicología, 19(1), 85-92.

Sánchez-Sosa, J.J. \& Alvarado, A.S. (2008). A beha- vioral self-recording procedure in the management of breast cancer: A field test with disadvantaged participants. Revista Mexicana de Análisis de la Conducta, 34(2), 313- 331.

Sánchez-Sosa, J.J. \& González-Celis, A.L. (2006). Evaluación de la calidad de vida desde la perspectiva psicológica. En: V. E. Caballo (Dir.) Manual para la Evaluación Clínica de los Trastornos Psicológicos (pp. 473-492). Madrid: Psicología Pirámide.

Secretaría de Salud (2003) RHNM. Compendio Mortalidad/Morbilidad CD.

Seitz, D. C. M., Besier, T. \& Goldbeck, L. (2009). Psychosocial interventions for adolescent cancer patients: A systematic review of the literature. Psycho-Oncology, 18(7), 683-690.

Sperry, L. (2010) Breast cancer, depression, culture, and marital conflict. The Family Journal, 18(1), 62-65.

Suinn, R.M. \& Richardson, F. (1971). Anxiety management training: A non specific behavior therapy program for anxiety control. Behavior Therapy, 2(4), 498-511.

Tamblay, A. \& Bañados, B. (2004). Psicooncología del cáncer de mama. Tesis de licenciatura. Universidad de Chile.

Thornton, L. M., Andersen, B. L., Blakely, W. P. (2010). The pain, depression, and fatigue symptom cluster in advanced breast cancer: Covariation with the hypothalamic-pituitary-adrenal axis and the sympathetic nervous system. Health Psychology, 29(3), 333-337.

Trull, T. \& Phares, J. (2003). Psicología Clínica (pp. 373404). México: Thompson.

Vandenberghe, L. (2008). Culture-sensitive functional analytic psychotherapy. The Behavior Analyst, 31(1), 67-79.

Velikova, G., Awad, N., Coles-Gale, R., Wright, E. P., Brown, J. M. \& Selby, P. J. (2008). The clinical value of quality of life assessment in oncology practice: A qualitative study of patient and physician views. Psycho-Oncology, 17(7), 690698.

Zimmermann, T., Heinrichs, N. \& Baucom, D. H. (2007). "Does one size fit all?" Moderators in psychosocial interventions for breast cancer patients: A meta-analysis. Annals of Behavioral Medicine, 34(3), 225-239. 